Article

\title{
A Hybrid Speller Design Using Eye Tracking and SSVEP Brain-Computer Interface
}

\author{
Malik M. Naeem Mannan ${ }^{1}{ }^{\circledR}$, M. Ahmad Kamran ${ }^{1}\left(\mathbb{D}\right.$, Shinil Kang ${ }^{2,3}$, Hak Soo Choi ${ }^{1,4}$ \\ and Myung Yung Jeong ${ }^{1, *}$ \\ 1 Department of Cogno-Mechatronics Engineering, Pusan National University, 2 Busandaehak-ro, 63 Beon-gil, \\ Geumjeong-gu, Busan 609-735, Korea; naeem@pusan.ac.kr (M.M.N.M.); malik@pusan.ac.kr (M.A.K.); \\ HCHOI12@mgh.harvard.edu (H.S.C.) \\ 2 National Center for Optically-Assisted Ultrahigh-Precision Mechanical Systems, Yonsei University, \\ Seoul 03722, Korea; snlkang@yonsei.ac.kr \\ 3 School of Mechanical Engineering, Yonsei University, Seoul 03722, Korea \\ 4 Division of Hematology/Oncology, Department of Medicine, Beth Israel Deaconess Medical Center and \\ Harvard Medical School, Boston, MA 02115, USA \\ * Correspondence: myjeong@pusan.ac.kr
}

Received: 31 December 2019; Accepted: 5 February 2020; Published: 7 February 2020

\begin{abstract}
Steady-state visual evoked potentials (SSVEPs) have been extensively utilized to develop brain-computer interfaces (BCIs) due to the advantages of robustness, large number of commands, high classification accuracies, and information transfer rates (ITRs). However, the use of several simultaneous flickering stimuli often causes high levels of user discomfort, tiredness, annoyingness, and fatigue. Here we propose to design a stimuli-responsive hybrid speller by using electroencephalography (EEG) and video-based eye-tracking to increase user comfortability levels when presented with large numbers of simultaneously flickering stimuli. Interestingly, a canonical correlation analysis (CCA)-based framework was useful to identify target frequency with a $1 \mathrm{~s}$ duration of flickering signal. Our proposed BCI-speller uses only six frequencies to classify forty-eight targets, thus achieve greatly increased ITR, whereas basic SSVEP BCI-spellers use an equal number of frequencies to the number of targets. Using this speller, we obtained an average classification accuracy of $90.35 \pm 3.597 \%$ with an average ITR of $184.06 \pm 12.761$ bits per minute in a cued-spelling task and an ITR of $190.73 \pm 17.849$ bits per minute in a free-spelling task. Consequently, our proposed speller is superior to the other spellers in terms of targets classified, classification accuracy, and ITR, while producing less fatigue, annoyingness, tiredness and discomfort. Together, our proposed hybrid eye tracking and SSVEP BCI-based system will ultimately enable a truly high-speed communication channel.
\end{abstract}

Keywords: Brain-computer interface; electroencephalography; eye tracker; hybrid BCI; canonical correlation analysis; steady-state visual evoked potentials; information transfer rate

\section{Introduction}

A brain-computer interface (BCI) provides a direct line of communication between a human brain and a computer by converting physiological signals into commands for the control of external devices [1-5]. BCIs are designed mainly to provide an alternative means of communication for people with severe motor disabilities [6-9]. Among a number of techniques, electroencephalography (EEG) is the most popular brain-imaging method for $\mathrm{BCI}$ implementation due to its noninvasive nature, low cost, portability and high temporal resolution [9-13]. In the literature, several BCI systems have been developed by using EEG signals, including [14] event-related desynchronization/synchronization 
(ERD/ERS) [3], steady-state visually evoked potentials (SSVEPs) [8,15], event-related potentials (ERPs) [16,17], and slow cortical potentials (SCPs) [18]. Among these, SSVEP-based BCIs are the most practical, because they support a large number of output commands and require little training time [19-27]. Recently, the SSVEP-based BCI has attracted increasing attention due to its high rate of communication and lack of any significant training requirement compared with other $\mathrm{BCI}$ systems [28-31]. Users of an SSVEP-based BCI are presented with a set of visual targets that are associated with possible characters, each of which flickers at a different, fixed frequency [32]. In an SSVEP BCI, target character/command where user is looking at is decoded by using corresponding SSVEP responses.

The use of various SSVEP-based BCI spellers for high classification accuracy as well as high communication rates has been proposed. Bin et al. reported, based on a six-target system, an information transfer rate (ITR) of 58 bits/min with an average accuracy of 95.3\% [33], while Nakanishi et al. achieved an average accuracy and ITR of $92.78 \%$ and $91.68 \mathrm{bits} / \mathrm{min}$ with a 12-target system [34] and $91.35 \%$ and 166.91 bits/min with a 32-target system [35]. Yin et al., utilizing a 36-target SSVEP-based BCI speller, reported an ITR of $41.08 \mathrm{bits} / \mathrm{min}$ with an accuracy greater than $85 \%$ [36]; later on, by using hybrid P300 and SSVEP scores, they were able to achieve an average accuracy of $95.18 \%$ with an ITR of $50.41 \mathrm{bits} / \mathrm{min}$ [22]. Chen et al. reported an ITR of $105 \mathrm{bits} / \mathrm{min}$ for a 45 -target BCI speller [27]; more recently, they introduced a high-speed spelling system with an average ITR of $267 \mathrm{bits} / \mathrm{min}$ and an average accuracy of $91.04 \%$ [28]. Nakanishi et al. developed a task-related component analysis technique to develop a high speed speller with an average accuracy of $89.93 \%$ and an average ITR of 325.33 bits/min [29]. In a recent study, Maye et al. proposed a multi-target SSVEP-based BCI system that only uses single flickering stimulus [37].

The stimulation frequencies ranging between 5 and $90 \mathrm{~Hz}$ could be used to elicit SSVEPs, but only few frequencies could be used due to the technological constraints of the current systems [38]. For instance, the conventional frequency coding method can only generate specific frequencies due to the limitation posed by the monitor refresh rate [39]. For example, a $60 \mathrm{~Hz}$ refresh rate monitor can only generate frequencies that are integer divisible of 60 , e.g., $60 / 2=30,60 / 3=20$, and $60 / 4=15$. Furthermore, Muller and co-authors showed that the detection of SSVEPs can be enhanced by using the harmonic components of the fundamental frequencies that are used to elicit SSVEPs, and, therefore, the use of harmonic frequencies should be avoided while designing SSVEP BCIs [40]. This further limit the available frequencies for a practical BCI system. For example, using $10 \mathrm{~Hz}$ as a fundamental stimulus frequency restricts the use of 20 and $30 \mathrm{~Hz}$ frequencies, since these are the harmonic components of $10 \mathrm{~Hz}$. Moreover, many studies have shown that low frequency stimuli's (4-12 Hz) can elicit strongest SSVEP responses; therefore, for high detection rates, stimulus frequencies should be selected from a low frequency range [41-43]. In the past, many researchers have proposed different stimuli designs to tackle these limitations and restrictions $[28,44-46]$. These methods include phase coding techniques [44,45], dual frequency stimuli [47,48], variable frame rate stimuli [49], multiple frequency sequential coding [50], amplitude modulation techniques [51,52], intermodulation frequencies [38,53,54], varying duty cycles [41,55], interpolation techniques [39], and joint frequency and phase modulation [28], but these techniques also have limitations. For instance, the number of phase lags are also dependent on the number of frames of the stimulus frequency in the phase tagging method, consequently resulting in a limited number of targets [38]. In the case of multiple frequency coding techniques, the number of targets increases as the cycle period increases, and the classification time window is proportional to the amount of stimulation frequencies that further decreases the overall BCI performance $[38,50]$. Furthermore, it has also been shown that low-frequency stimuli can cause photosensitivity-based epileptic seizures and high-levels of visual fatigue and discomfort, especially when presented with large number of targets and longer periods [21,43]. Many studies have used high-frequency stimuli and variable duty cycles to reduce visual fatigue and discomfort but at the cost of a decrease in the performance of SSVEP-based BCIs [21,43,56-58]. A few authors have also combined other EEG signals, e.g., P300, with SSVEP to overcome these limitations [22,59-63]. For instance, a 
64 target BCI system was developed by using eight SSVEP frequencies and the P300 paradigm [22]. Though the number of targets can be increased by using only few frequencies, the incorporation of P300 increases the complexity of the task, which can also cause fatigue and a reduction in the classification accuracies.

On the other hand, researchers are combing several other modalities with EEG to enhance the performances of the current techniques. Recently, a bimodal approach that combined SSVEP with Electromyography (EMG) was developed to generate a 60 target speller with only 15 frequencies [64]. The speller was divided into four equal sections. The researchers instructed subjects to make fists (0-3) to identify the target section (1-4) and SSVEPs to detect target frequency. The data in this paper showed an average accuracy of $85.8 \%$ and an average ITR of 90.9 bits/min. Furthermore, eye tracking-based assistive technologies are emerging as an alternative tool to BCIs $[65,66]$. The development of modern camera systems and the increase of computing power has enabled the gathering of eye tracking data in real time, enabling the use of gaze as a control method for people with disabilities [67-73]. A comparison study of $\mathrm{BCI}$, eye tracking, and electrooculography interfaces reported that participants found the BCI to be the easiest to use and eye tracking to be the least tiring [65]. They also showed that an SSVEP-based BCI performed better than an eye tracking-based speller when targets are densely located and small in size. Recently, eye tracking has been combined with EEG to remove ocular artifacts from EEG signals [74,75] and to develop hybrid BCI systems [65,76-81]. All these studies have shown improved performance as compared to EEG only, as well as the feasibility of combining EEG with eye tracking.

This paper proposes a combined EEG/eye tracking system for high-speed speller implementation in order to overcome the limitations described above; specifically (a) to improve practicality, especially on a computer screen (where only a limited number of flickering targets could be reliably implemented), and (b) a better user experience. Furthermore, the proposed speller identifies forty-eight targets by using only six frequencies by dividing speller into eight sub-matrices with six targets each and does so with an improved classification accuracy and an increased ITR. Eye-tracker data are employed to identify the target sub-matrix, while EEG data are used to identify the target frequency of the SSVEP. Moreover, the proposed speller reduces users' visual discomfort, tiredness, annoyingness and fatigue, allowing for longer-duration use of the speller without any performance decrement; in contrast, a conventional BCI-speller (from now on referred to as a basic SSVEP speller) causes high fatigue and tiredness, which is a major problem that is responsible for performance decrement $[43,52,82,83]$. The proposed system is superior to basic SSVEP BCI-speller performances in terms of items classified, classification accuracy, and ITR. The comparison in the performances of the proposed, basic, and hybrid speller reveals the improved performance of the proposed hybrid approach. The significance of the performance improvement is statistically validated. In this study, a canonical correlation analysis (CCA)-based method is used to identify the target SSVEP frequency. In contrast to previous studies [34,83-85], the probability of misclassification of the targets with CCA is largely decreased by using six frequencies in the proposed hybrid approach. The methodological framework of the proposed system makes a truly high-speed communication channel possible.

\section{Materials and Methods}

\subsection{Materials}

\subsubsection{Proposed Hybrid SSVEP- and Eye-Tracking-Based Speller}

The proposed hybrid eye-tracking and SSVEP BCI-based communication system operates as follows. While the user gazes at a target character flickering at a certain frequency, the SSVEP responses of the recorded EEG data are estimated and used as feature vectors. The target character that the user gazes at is identified by using the proposed framework illustrated in Figure 1a, which simply finds the frequency with the largest SSVEP response and locates the target box with the help of eye-tracking data. Once the target frequency and box are identified, the target letter is typed. 
(a)

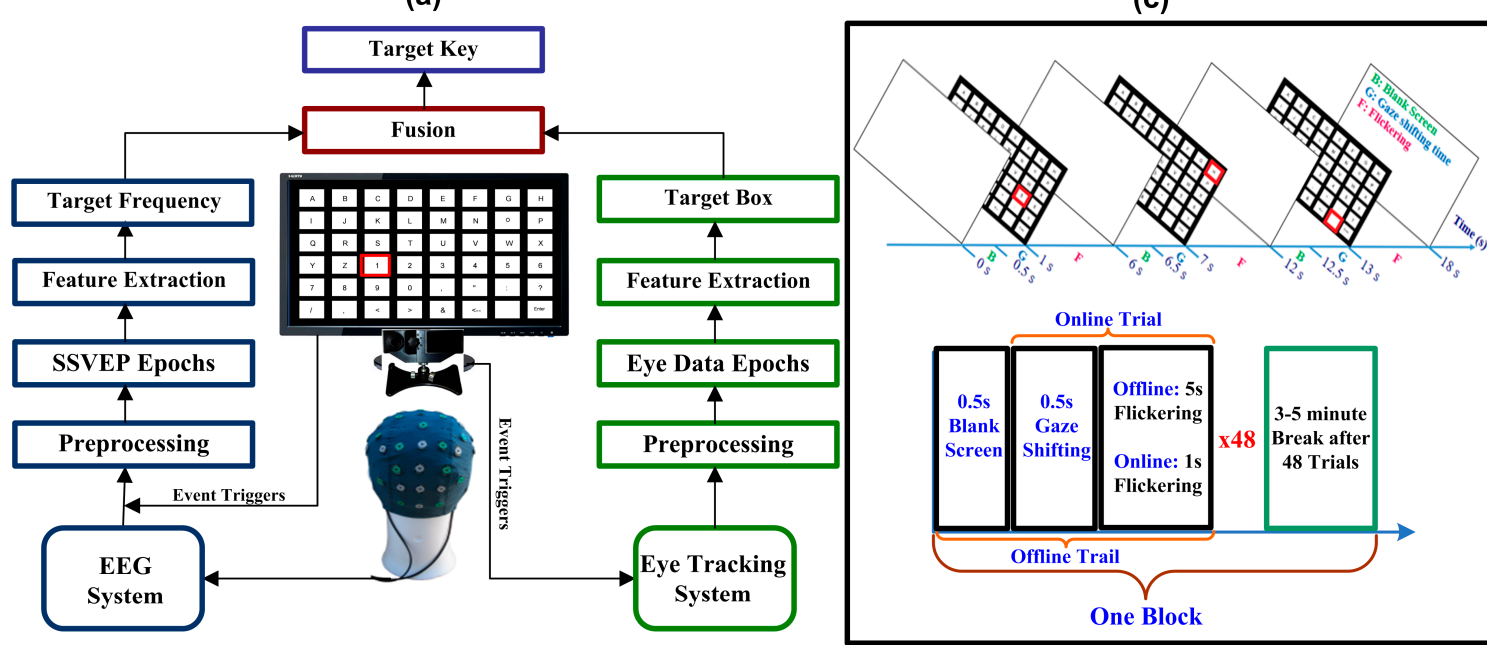

(b)

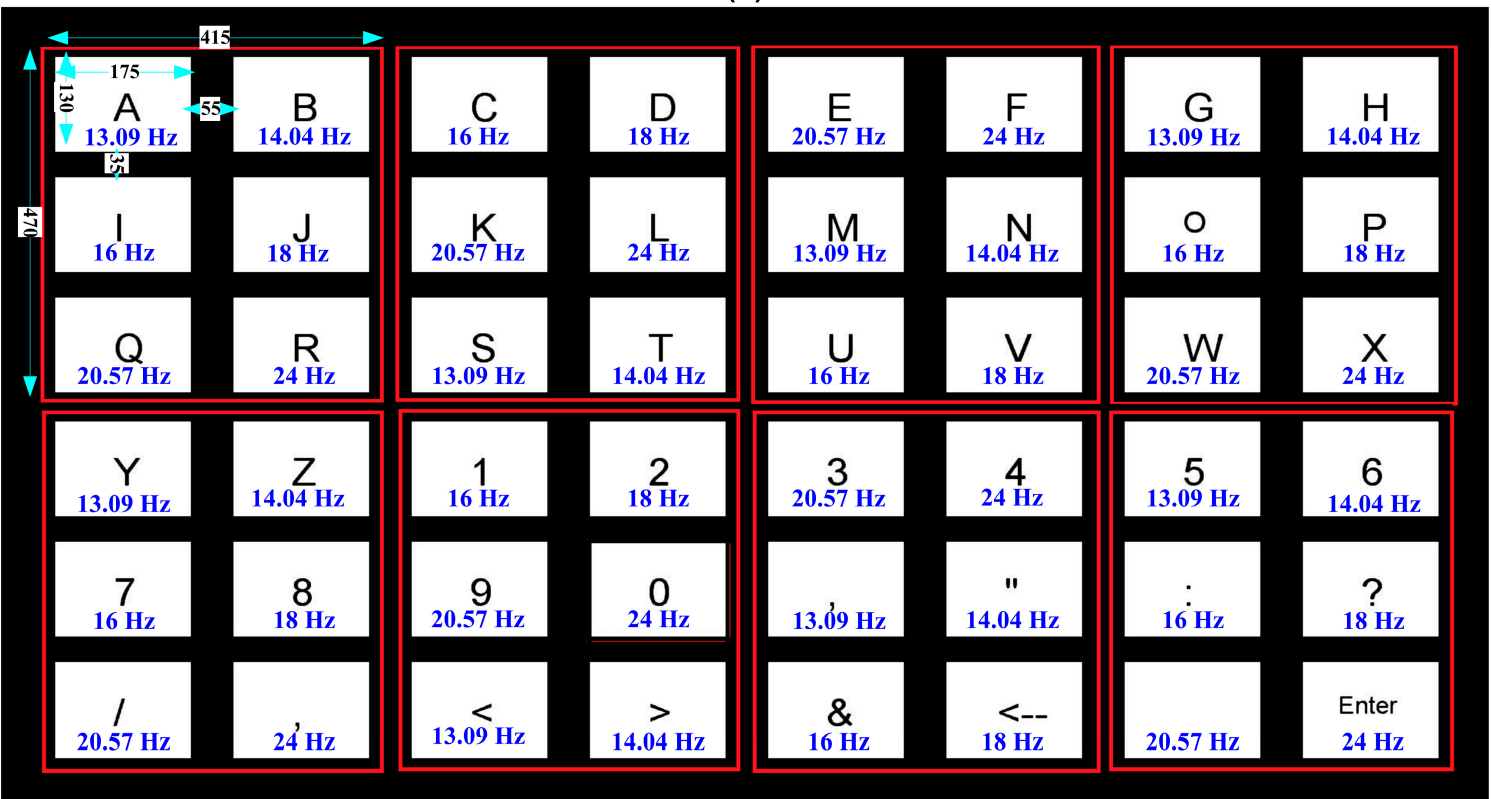

Figure 1. (a) Schematic diagram of the proposed framework. (b) Schematic diagram of the proposed speller with frequency of each target. Sizes were described in pixels. (c) Experimental paradigm.

\subsubsection{Participants}

Twenty healthy participants (male: 16, female: 4, aged $24-46$ years) participated in this study. All had normal or corrected-to-normal vision. Only four had previous experience with an SSVEP-based BCI; the others were naïve to it. The experimental protocol was approved by the Institutional Review Board of Pusan National University. The experiment was conducted in accordance with the ethical guidelines established by the Institutional Review Board of Pusan National University and the Declaration of Helsinki. Each participant was asked to sign a written informed consent after being completely informed about the nature and purpose of the study. Each participant completed an offline experiment before performing the online experiments. To analyze and compare the effect of fatigue, tiredness, annoyingness, and discomfort, each participant performed experiments for a basic SSVEP speller on different days with a minimum gap of three days. During the experiments, all of the participants were seated in a comfortable armchair at a viewing distance of about $70 \mathrm{~cm}$ from the monitor. The experiment was conducted in a confined room with dim lighting to avoid environmental disturbance. 


\subsubsection{Experimental Procedure}

The new 48-target BCI speller, the core of the proposed high-speed communication system, uses only six frequencies. As shown in Figure $1 \mathrm{~b}$, the user interface is a $6 \times 8$ stimulation matrix containing 48 characters including the 26-letter Roman alphabet, 10 digits, and 12 other symbols. The matrix speller is divided into eight equal $3 \times 2$ sub-matrices, each containing six characters. Each character in each sub-matrix is assigned a different, fixed frequency. The eye tracker is used to identify the corresponding sub-matrix, specifically by tracking the user gaze, while EEG data are analyzed to identify the target frequency. A 24-inch LCD monitor (Asus, $144 \mathrm{~Hz}$ refresh rate) with a resolution of $1920 \times 1080$ pixels was used to present the speller. In this study, the frequencies used to flicker the targets were integer divisors of the monitor refresh rate, i.e., $144 / 11=13.0909,144 / 10=14.40$, $144 / 9=16.00,144 / 8=18.00,144 / 7=20.5714$ and $144 / 6=24.00 \mathrm{~Hz}$. A stimulus program was developed by using Psychophysics Toolbox Version 3 with MATLAB to generate visual stimuli. Event triggers were sent from the parallel port of the computer to the both EEG and eye tracking systems.

\subsubsection{Offline Experiment}

For each participant, the offline experiment with the 48-key speller consisted of three blocks. In each block, all 48 targets were presented randomly. Thus, each participant had three trials per character for a total of $144(3 \times 48)$ trials. Each trial, of 6 s duration, started with a visual cue (a red square indicating a target stimulus) appearing on the screen for $0.5 \mathrm{~s}$. The participants were advised to move their gaze to the target character as quickly as possible within the cue period. All the target characters started to flicker for $5 \mathrm{~s}$ right after the cue offset. Before the next stimulus, the screen was blank for $0.5 \mathrm{~s}$ after each trial. The subjects were instructed to avoid blinks during flickering periods. There was a rest for few minutes after each block. Figure $1 \mathrm{c}$ describes the experimental paradigm for the offline and online experiments.

\subsubsection{Online Experiment}

In the online experiment, each trial was of only $1.50 \mathrm{~s}$ duration, including $0.5 \mathrm{~s}$ for gaze shifting and $1 \mathrm{~s}$ for stimulus flickering. The experiment was conducted in two stages, i.e., training and testing stage. There were three blocks in the training stage, each consisting of 48 trials to familiarize the subjects with online layout of the system. The testing stage included a cued-spelling task and a free-spelling task with three blocks each. In the cued-spelling task, targets were presented with a red box indicating a cue for the target character, whereas no cue was used in the free-spelling task. Each block in the cued-spelling task consisted of 48 trials. Auditory (a sound beep at the start of trial) and visual (red box on target stimuli) feedback was provided to the participants in real time. As soon as the target was classified by the online data analysis program, the identified target was typed into the text input field. In the free-spelling task, all participants were asked to input a 15-character sentence ("I LIVE IN KOREA") without any cues. There was a 3 to 5 -min break between two consecutive blocks.

\subsubsection{Control Conditions}

After completing the experiments with the proposed speller, each participant performed experiments with basic and hybrid spellers from the literature. Each participant was given break of at least three days between each experiment.

\section{Basic Speller}

In this study, a forty-eight-target conventional speller with only SSVEPs was also implemented to compare the performance of the proposed system. The speller was developed by using Psychophysics Toolbox Version 3 with MATLAB. A conventional sinusoidal frequency coding scheme was used to generate flickering stimuli [86]. The frequency range for basic speller was 7-16.4 Hz (around alpha band) with a step size of $0.2 \mathrm{~Hz}$. 


\section{Hybrid EEG-Eye Tacking}

In this study, the proposed hybrid approach was also compared with a previously developed hybrid mental spelling system [81]. The basic idea of [81] was to divide the speller into three parts, i.e., left, middle, and right. In this sense, the misclassification of the SSVEPs could be reduced to improve the classification accuracies and ITR of the system. The number of frequencies that were used in this speller were equal to the number of targets. We implemented this speller with forty-eight targets with frequencies ranging from 7 to $16.4 \mathrm{~Hz}$ (around alpha band) with a step size of $0.2 \mathrm{~Hz}$.

\subsubsection{Questionnaire}

In addition to conventional comparison of classification accuracies and ITR, each subject also completed a questionnaire about how they felt after the experiment. The questionnaire included questions about the previous experience with BCIs, as well as their discomfort, fatigue and tiredness after the experiment. For each participant, the experiments with proposed and control conditions were performed on different days to counterbalance the settings.

\subsubsection{EEG Recordings}

EEG data were recorded using a gUSBAmp with a 16-channel active electrode system that was developed by g.tec Medical Engineering, $\mathrm{GmbH}$ (Austria). Eight electrodes positioned over the parietal and occipital areas (PO7, PO3, POz, PO4, PO8, O1, Oz, and O2) were used to record the SSVEPs with a ground electrode FPz and reference electrode on right ear. All of the data were sampled at a rate of $1200 \mathrm{~Hz}$. All the electrodes were positioned according to international 10-20 system. The impedance of all of the electrodes was reduced to below $1 \mathrm{kohm}$.

\subsubsection{Eye-Tracker Recordings}

The eye movements were recorded with a video eye-tracking system (Eyelink 1000, developed by SR Research Ltd., Ottawa, ON, Canada). The sampling rate was $250 \mathrm{~Hz}$. A velocity threshold of $30^{\circ} / \mathrm{s}$ was used to define the saccades; the acceleration threshold and minimum deflection threshold were $8000^{\circ} / \mathrm{s}^{2}$ and $0.1^{\circ}$, respectively. The eye tracker was calibrated for each participant.

\subsection{Methods}

In this study, eye tracking data and the CCA algorithm were used to identify the target sub-matrix and frequency of SSVEPs, respectively. Before analyzing data, eye tracking and EEG data were synchronized by using event triggers sent to both systems through the parallel port of the computer. In the offline and online experiments, data epochs were extracted according to event triggers that were produced by the MATLAB program. Given the latency delay in the visual system, the data epochs for the experiments were extracted in [0.14 s $5.14 \mathrm{~s}$ ] and [0.14 s $1.14 \mathrm{~s}$ ], respectively (time 0 indicated stimulus onset) [34]. To remove the common power line noise in EEG data, a notch filter of $50 \mathrm{~Hz}$ was applied to the data recording. In both the offline and online experiments, all of the epochs were first down-sampled to $300 \mathrm{~Hz}$ and then band-pass-filtered from 12 to $110 \mathrm{~Hz}$. All the processing and analysis was performed in MATLAB.

\subsubsection{Sub-Matrix Detection}

In this study, eye tracking data were used to identify the target sub-matrix in the proposed system. Eye tracker data were stored as pixels. Data epochs that were extracted using event triggers were used in this analysis. The mean of the gaze-direction data from each epoch was calculated as a feature to classify target sub-matrices. 


\subsubsection{SSVEP Detection}

CCA is a method for the extraction of similarities between two data sets [34,87]. CCA was first used in BCI studies by Lin et al. to detect SSVEP frequencies [88]. Considering two multidimensional variables $X, Y$ and their linear combinations $x=X^{T} w_{x}$ and $y=Y^{T} w_{y}$, CCA finds the weight vectors, $w_{x}$ and $w_{y}$, that maximize the correlation between $x$ and $y$ by solving the problem:

$$
\rho(x, y)=\max _{w_{x}, w_{y}} \frac{E\left[x^{T} y\right]}{\sqrt{E\left[x^{T} x\right] E\left[y^{T} y\right]}}=\max _{w_{x}, w_{y}} \frac{E\left[w_{x}{ }^{\mathbf{T}} X \mathbf{Y}^{\mathbf{T}} \mathbf{w}_{y}\right]}{\sqrt{E\left[w_{x}^{\mathbf{T}} X X^{\mathbf{T}} \mathbf{w}_{x}\right] E\left[w_{y}^{\mathbf{T}} Y \mathbf{Y}^{\mathbf{T}} \mathbf{w}_{y}\right]}}
$$

The maximum of $\rho$ with respect to $w_{x}$ and $w_{y}$ is the maximum canonical correlation. Projections onto $w_{x}$ and $w_{y}$ are called canonical variants. Here, $X$ refers to a set of multi-channel EEG signals, and $Y$ refers to the set of reference signals that have the same length as $X$. In SSVEP detection, the reference signals $Y_{k} \in \mathfrak{R}^{2 N_{h} \times N}$ are set as

$$
Y_{n}=\left[\begin{array}{l}
\sin \left(2 \pi f_{k} t\right) \\
\cos \left(2 \pi f_{k} t\right) \\
\vdots \\
\sin \left(2 \pi N_{h} f_{k} t\right) \\
\cos \left(2 \pi N_{h} f_{k} t\right)
\end{array}\right], t=\left[\frac{1}{f_{s}}, \frac{2}{f_{s}}, \cdots, \frac{N}{f_{s}}\right]
$$

where $f_{k}$ is the stimulation frequency, $f_{s}$ is the sampling frequency, $N_{h}=3$ is the number of harmonics, and $N$ is the number of sample points. To recognize the frequency of the SSVEPs, CCA calculates the canonical correlation $\rho_{k}$ between the multi-channel EEG signals $X$ and the reference signals at each stimulus frequency $Y_{k}$. The frequency of the reference signals with the maximal correlation is then selected as the frequency of the SSVEPs.

Once the target sub-matrix and frequency were identified, the corresponding character was selected as an output.

\subsubsection{Performance Evaluation}

Classification accuracy and ITR were separately calculated for the offline and online experiments. The method for the calculation of ITR (in bits per minute, bpm) was [35]:

$$
I T R=\frac{1}{T}\left[\log _{2} M+P \log _{2} P+(1-P) \log _{2}\left(\frac{1-P}{M-1}\right)\right] \times 60
$$

where $M$ represents the total targets (i.e., 48 in this study), $P$ represents the classification accuracy, and $T$ represents the average selection time. In the offline experiments, the optimal BCI performance to calculate classification accuracy and ITR was estimated by using time windows between 1 and $4 \mathrm{~s}$ with an increment of $0.5 \mathrm{~s}$. For the online experiment, the accuracy and ITR were calculated by using results obtained from the online data analysis program. In this study, the time windows that were used to calculate ITR for both the offline and online experiments also included the gaze shifting time (i.e., $T=0.5 \mathrm{~s}+1 \mathrm{~s}=1.5 \mathrm{~s}$ ).

\section{Results}

In this paper, we propose a hybrid strategy to increase user comfort and to achieve high eye tracking and SSVEP-BCI-based speller classification accuracy and ITR.

\subsection{Offline Data Analysis}

To verify that the proposed framework performed better, the proposed approach was analyzed for the proposed 48-target speller in an offline experiment. Furthermore, the performance of the proposed 
speller was compared with the performance of a previously developed basic BCI-speller with SSVEPs only and hybrid EEG and eye tracking-based speller systems. Figure 2a shows a comparison of the average classification accuracies that were achieved by the proposed framework (black line), basic speller (blue line), and hybrid speller (orange line) for all subjects for epoch lengths ranging from 1 to $4 \mathrm{~s}$ with a fixed increment of $0.5 \mathrm{~s}$; Figure $2 \mathrm{~b}$ shows the corresponding average ITRs achieved by the proposed framework (black line), basic speller (blue line), and hybrid speller (orange line) with different epoch lengths. It could be seen that the accuracies and ITRs were higher for the proposed hybrid approach than those for the basic BCI-speller and the hybrid speller. Moreover, the significance of this performance improvement was statistically validated by using Mann-Whitney $U$ test. The results of this statistical analysis verified that the performance improvement by the proposed hybrid approach was significantly higher than basic BCI-speller and hybrid speller for all time windows with $p<0.001$. Additionally, in order to evaluate the discomfort, annoyingness, eye fatigue, and tiredness that was caused by the proposed, basic and hybrid speller systems, each subject was asked to fill in a questionnaire concerning his experience in the use of all speller systems after the experiments. The results of these questionnaire are listed in Table 1 . It can be seen that $60 \%$ of the subjects felt a low level of annoyance due to flickering when using the proposed speller, whereas $55 \%$ of the subjects were highly annoyed when using the basic BCI-speller and the hybrid speller. Moreover, none of the subjects felt a high level of eye fatigue after using the proposed BCI-speller, while $65 \%$ of the subjects were highly fatigued after using both of the previously developed spellers. Furthermore, all the subjects were also asked about the overall tiredness they felt after using all these spellers. It is noteworthy that none of the subjects felt tiredness above a medium level after using the proposed speller, whereas $75 \%$ of the subjects were highly tired after using the other spellers. According to the results, all of the subjects were significantly more comfortable using the proposed speller system as compared with the spellers used in previous BCI studies [34,39]. Thus, the proposed speller could be implemented as a more comfortable and easy-to-use mode for practical and clinical applications, e.g., patients in locked-in state [89]. Since the average accuracy with the proposed speller was relatively higher $(>89.03 \%)$ with all of the epoch lengths, the highest ITR was obtained with the shortest epoch length. According to Nakanishi et al. [35] and Equation (3) when using longer-length data, a minor increase in the classification accuracy leads to a significant decrease of ITR. For example, compared with $1 \mathrm{~s}$ data length, classification accuracy increased by $3.65 \%$ when using the $2 \mathrm{~s}$ data length $(89.02 \%$ vs. $92.67 \%)$; however, the ITR dropped from 179.60 to $144.32 \mathrm{bpm}$. Correspondingly, in an online system that used the proposed framework, the $1 \mathrm{~s}$ epoch length was found to be optimal for the achievement of a high ITR, and there was no overlapping in the epochs. Figure 2c shows the percentage of the correct trials that were identified in each block by the proposed framework for each subject. Table 2 shows all of the subjects' classification accuracies and ITR for an epoch length of $1 \mathrm{~s}$.

Table 1. Results from the questionnaires.

\begin{tabular}{|c|c|c|c|c|c|c|c|c|c|c|c|c|c|}
\hline \multirow[t]{2}{*}{ Speller } & \multicolumn{2}{|c|}{$\begin{array}{l}\text { Experience with } \\
\text { SSVEP BCI }\end{array}$} & \multicolumn{3}{|c|}{ Flickering Annoying } & \multicolumn{3}{|c|}{ Eye Fatigue } & \multicolumn{5}{|c|}{ Level of Tiredness } \\
\hline & Yes & No & Low & Medium & High & Low & Medium & High & 1 & 2 & 3 & 4 & 5 \\
\hline Proposed & & & 12 & 8 & 0 & 12 & 8 & 0 & 7 & 10 & 3 & 0 & 0 \\
\hline Basic & 4 & 16 & 0 & 9 & 11 & 0 & 7 & 13 & 0 & 0 & 5 & 10 & 5 \\
\hline $\begin{array}{c}\text { Hybrid EEG } \\
\text { and eye } \\
\text { tracking }\end{array}$ & & & 0 & 8 & 12 & 0 & 8 & 12 & 0 & 0 & 4 & 11 & 5 \\
\hline
\end{tabular}

The numbers represent number of subjects. The level of tiredness was evaluated on a scale of 1 to $5: 1=$ not tired; 2 $=$ little tired; $3=$ moderately tired; $4=$ tired; and $5=$ highly tired. 
(a)

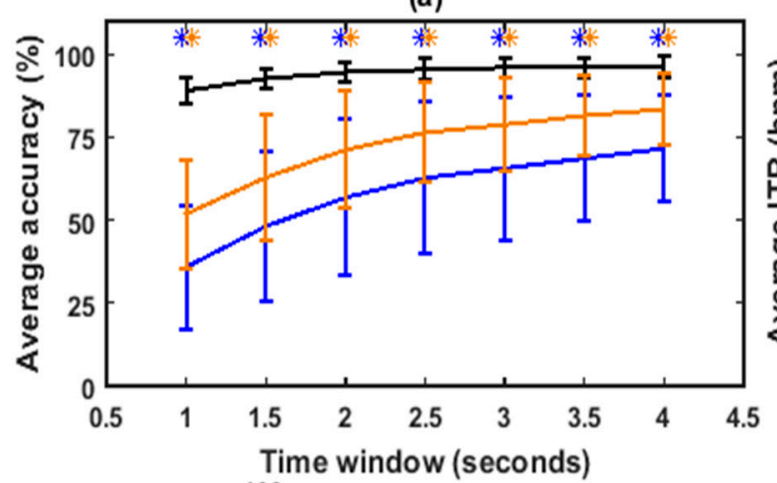

(b)

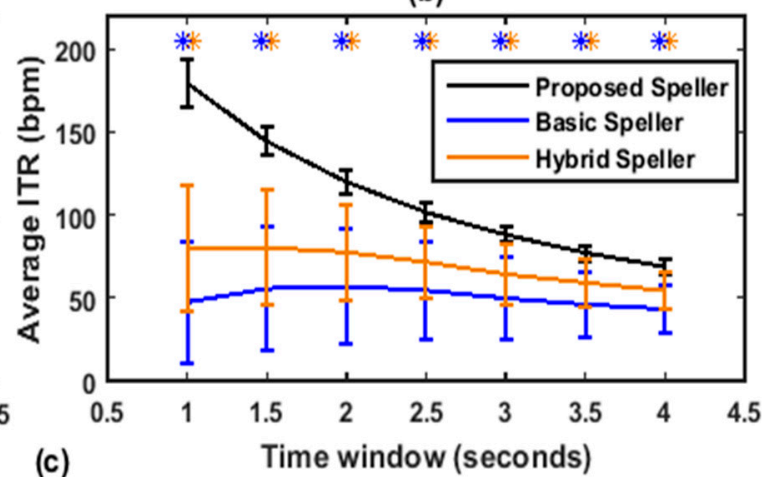

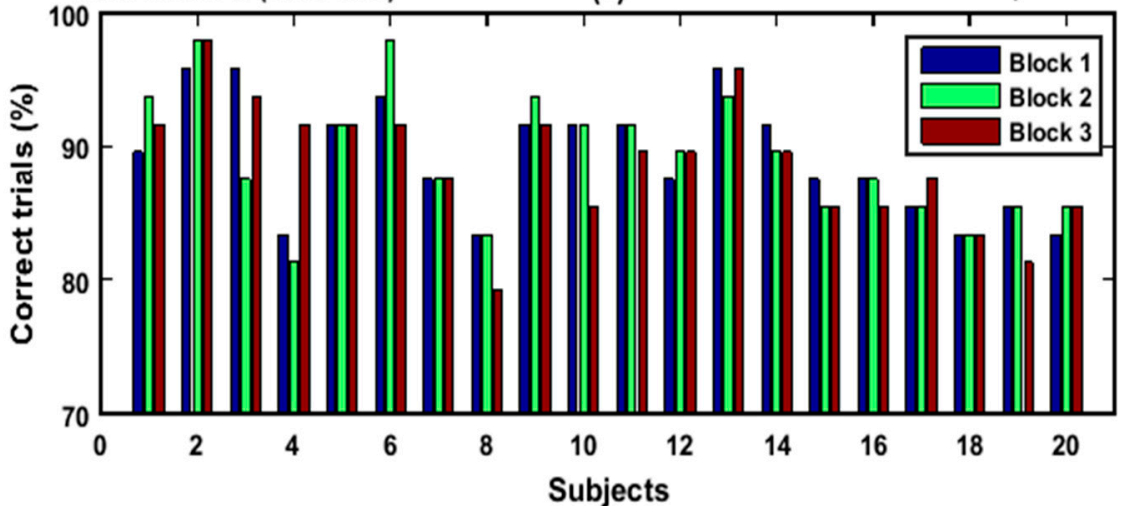

Figure 2. Performance comparison and evaluation of the proposed speller, the basic steady-state visually evoked potential brain-computer interface (SSVEP BCI)-speller, and the hybrid electroencephalography (EEG)-eye tracking-based speller for all subjects with different time windows during the offline experiment: proposed speller (black line), basic speller (blue line) and hybrid speller (orange line). (a) Average classification accuracies, (b) average information transfer rate (ITR), and (c) percentage of correct trials identified in each block for each subject. In $(\mathbf{a}, \mathbf{b})$, error bars indicate standard deviation. The asterisks indicate a significantly improved performance by the proposed speller $\left({ }^{*} p<0.001\right)$.

Table 2. Classification accuracy and information transfer rate of proposed framework with $1 \mathrm{~s}$ epoch lengths in offline experiment.

\begin{tabular}{ccc}
\hline Sub & Classification Accuracy $\mathbf{( \% )}$ & Information Transfer Rate $\mathbf{( b p m )}$ \\
\hline $\mathbf{1}$ & 91.67 & 188.34 \\
$\mathbf{2}$ & 97.22 & 209.89 \\
$\mathbf{3}$ & 92.36 & 190.84 \\
$\mathbf{4}$ & 85.42 & 167.03 \\
$\mathbf{5}$ & 91.67 & 188.34 \\
$\mathbf{6}$ & 95.14 & 201.38 \\
$\mathbf{7}$ & 87.50 & 173.88 \\
$\mathbf{8}$ & 81.94 & 156.02 \\
$\mathbf{9}$ & 89.58 & 180.96 \\
$\mathbf{1 0}$ & 92.36 & 190.85 \\
$\mathbf{1 1}$ & 90.97 & 185.84 \\
$\mathbf{1 2}$ & 88.89 & 178.59 \\
$\mathbf{1 3}$ & 95.14 & 201.38 \\
$\mathbf{1 4}$ & 90.28 & 183.40 \\
$\mathbf{1 5}$ & 86.11 & 169.28 \\
$\mathbf{1 6}$ & 86.81 & 171.58 \\
$\mathbf{1 7}$ & 86.11 & 169.28 \\
$\mathbf{1 8}$ & 83.33 & 160.35 \\
\hline
\end{tabular}


Table 2. Cont.

\begin{tabular}{ccc}
\hline Sub & Classification Accuracy (\%) & Information Transfer Rate (bpm) \\
\hline $\mathbf{1 9}$ & 84.03 & 162.57 \\
$\mathbf{2 0}$ & 84.72 & 164.78 \\
Mean & $\mathbf{8 9 . 0 3}$ & $\mathbf{1 7 9 . 6 0}$ \\
SD & 4.224 & $\mathbf{1 4 . 7 2 8}$ \\
\hline
\end{tabular}

\subsection{Online Data Analysis}

This study evaluated the proposed BCI speller while using two online cued-and free-spelling tasks. Table 3 lists the classification accuracy and ITR for all subjects cued-spelling tasks in the training and testing sessions. The average accuracy in the cued-spelling task in training session was $89.72 \%$, which resulted in an average ITR of $181.90 \mathrm{bpm}$ across all subjects. In the testing session, the average accuracy and ITR were $90.35 \%$ and $184.06 \mathrm{bpm}$, respectively. The online classification accuracy and ITR were slightly higher than those obtained in the offline experiment (accuracy: 89.03\% vs. 90.35\%; ITR: $179.60 \mathrm{bpm}$ vs $184.06 \mathrm{bpm}$; Tables 2 and 3). This could have been due to the familiarization with the proposed hybrid approach achieved by the subjects in the training sessions. Across individuals, the minimal and maximal ITR were $162.57 \mathrm{bpm}$ (subject 19) and $206.98 \mathrm{bpm}$ (subject 13), respectively. Table 4 shows the results of the free-spelling tasks. After some practice sessions for familiarization with the speller layout (without any cue), all of the subjects successfully completed the tasks. For subjects $7,11,15$ and 18 , the stimulus time was increased to $1.25 \mathrm{~s}$ to improve the classification accuracy; for subjects 4,8 and 19, the gaze-shifting time was increased to $1 \mathrm{~s}$ due to the difficulty in rapidly shifting their gaze. The mean ITR achieved by the system was $190.73 \mathrm{bpm}$ (minimum: $159.23 \mathrm{bpm}$ (subjects 4, 8 and 19); maximum: 212.31 (subjects 6, 10 and 13)). An average spelling rate of 35.79 characters per minute (cpm) was achieved by the proposed system with maximum of $39.11 \mathrm{cpm}$. The overall results showed that there were no significant differences in the ITR of the cued- and free-spelling tasks.

Table 3. Classification accuracy and information transfer rate for the online cued-spelling task.

\begin{tabular}{ccccc}
\hline \multirow{2}{*}{ Sub } & \multicolumn{2}{c}{ Training Session } & \multicolumn{2}{c}{ Testing Session } \\
\cline { 2 - 5 } & $\begin{array}{c}\text { Classification } \\
\text { Accuracy (\%) }\end{array}$ & $\begin{array}{c}\text { Information Transfer } \\
\text { Rate (bpm) }\end{array}$ & $\begin{array}{c}\text { Classification } \\
\text { Accuracy } \mathbf{( \% )}\end{array}$ & $\begin{array}{c}\text { Information Transfer } \\
\text { Rate (bpm) }\end{array}$ \\
\hline $\mathbf{1}$ & 89.58 & 180.97 & 88.19 & 176.21 \\
$\mathbf{2}$ & 95.13 & 201.38 & 95.13 & 201.38 \\
$\mathbf{3}$ & 90.97 & 185.84 & 92.36 & 190.85 \\
$\mathbf{4}$ & 87.50 & 173.88 & 86.81 & 171.57 \\
$\mathbf{5}$ & 92.36 & 190.85 & 93.06 & 193.42 \\
$\mathbf{6}$ & 95.83 & 204.15 & 95.83 & 204.15 \\
$\mathbf{7}$ & 88.19 & 176.22 & 89.58 & 180.97 \\
$\mathbf{8}$ & 86.80 & 171.57 & 86.11 & 169.28 \\
$\mathbf{9}$ & 92.36 & 190.85 & 93.06 & 193.42 \\
$\mathbf{1 0}$ & 93.75 & 196.02 & 93.06 & 193.42 \\
$\mathbf{1 1}$ & 88.89 & 178.58 & 91.67 & 188.33 \\
$\mathbf{1 2}$ & 91.67 & 188.33 & 92.36 & 190.85 \\
$\mathbf{1 3}$ & 95.83 & 204.15 & 96.53 & 206.98 \\
$\mathbf{1 4}$ & 88.89 & 178.58 & 91.97 & 185.84 \\
$\mathbf{1 5}$ & 85.42 & 167.02 & 87.50 & 173.88 \\
$\mathbf{1 6}$ & 90.97 & 185.84 & 90.28 & 183.39 \\
$\mathbf{1 7}$ & 85.42 & 167.02 & 86.81 & 171.57 \\
$\mathbf{1 8}$ & 86.81 & 171.57 & 86.11 & 169.29 \\
$\mathbf{1 9}$ & 82.64 & 158.19 & 84.03 & 162.57 \\
$\mathbf{2 0}$ & 85.42 & 167.02 & 87.50 & 173.88 \\
\hline Mean & $\mathbf{8 9 . 7 2}$ & $\mathbf{1 8 1 . 9 0}$ & $\mathbf{9 0 . 3 5}$ & $\mathbf{1 8 4 . 0 6}$ \\
$\mathbf{S D}$ & $\mathbf{3 . 7 8 8}$ & $\mathbf{1 3 . 2 9 8}$ & 3.597 & $\mathbf{1 2 . 7 6 1}$ \\
\hline
\end{tabular}


Table 4. Classification accuracy and information transfer rate for the online free-spelling task.

\begin{tabular}{|c|c|c|c|c|}
\hline Sub & $\begin{array}{c}\text { Trial Length (s) } \\
\text { (Gaze Shift + Stimulus) }\end{array}$ & $\begin{array}{l}\text { Total No. of Trials } \\
\text { (Correct/Incorrect) }\end{array}$ & $\begin{array}{l}\text { Spelling Rate } \\
(\mathrm{cpm})\end{array}$ & $\begin{array}{l}\text { Information Transfer } \\
\text { Rate (bpm) }\end{array}$ \\
\hline 1 & $1.5(0.5+1)$ & $45(41 / 4)$ & 36.44 & 186.34 \\
\hline 2 & $1.5(0.5+1)$ & $45(43 / 2)$ & 38.22 & 203.03 \\
\hline 3 & $1.5(0.5+1)$ & $45(43 / 2)$ & 38.22 & 203.03 \\
\hline 4 & $2.0(1+1)$ & $45(44 / 1)$ & 29.36 & 159.23 \\
\hline 5 & $1.5(0.5+1)$ & $45(43 / 2)$ & 38.22 & 203.03 \\
\hline 6 & $1.5(0.5+1)$ & $45(44 / 1)$ & 39.11 & 212.31 \\
\hline 7 & $1.75(0.5+1.25)$ & $45(43 / 2)$ & 32.76 & 174.02 \\
\hline 8 & $2.0(1+1)$ & $45(44 / 1)$ & 29.36 & 159.23 \\
\hline 9 & $1.5(0.5+1)$ & $45(43 / 2)$ & 38.22 & 203.03 \\
\hline 10 & $1.5(0.5+1)$ & $45(44 / 1)$ & 39.11 & 212.31 \\
\hline 11 & $1.75(0.5+1.25)$ & $45(44 / 1)$ & 33.52 & 181.98 \\
\hline 12 & $1.5(0.5+1)$ & $45(42 / 3)$ & 37.33 & 194.45 \\
\hline 13 & $1.5(0.5+1)$ & $45(44 / 1)$ & 39.11 & 212.31 \\
\hline 14 & $1.5(0.5+1)$ & $45(43 / 2)$ & 38.22 & 203.03 \\
\hline 15 & $1.75(0.5+1.25)$ & $45(44 / 1)$ & 33.52 & 181.98 \\
\hline 16 & $1.5(0.5+1)$ & $45(43 / 2)$ & 38.22 & 203.03 \\
\hline 17 & $1.5(0.5+1)$ & $45(42 / 3)$ & 37.33 & 194.45 \\
\hline 18 & $1.75(0.5+1.25)$ & $45(43 / 2)$ & 32.76 & 174.03 \\
\hline 19 & $2.0(1+1)$ & $45(44 / 1)$ & 29.36 & 159.23 \\
\hline 20 & $1.5(0.5+1)$ & $45(42 / 3)$ & 37.33 & 194.45 \\
\hline Mean & - & - & 35.79 & 190.73 \\
\hline SD & & & 3.47 & 17.849 \\
\hline
\end{tabular}

\section{Discussion}

User comfortability plays an important role in the performance of BCI systems. As discussed in previous studies, in basic BCI spellers, the flickering of a large number of frequencies causes eye fatigue and discomfort for patients, thus rendering concentration on specific targets difficult, especially after using the speller for long period of time $[43,52,82,83]$. In the past, the necessity of decoding a large number of frequencies remained the key obstacle to the improvement of BCI-based spelling systems' classification accuracy and ITR. Certainly, classification-accuracy and ITR shortcomings are precisely the problems that make the practical implementation of BCI spellers so difficult. However, recent advances in SSVEP-based BCIs have developed techniques and algorithms to overcome these obstacles. Several studies have proposed different stimulus design techniques to realize large number of targets with only few frequencies, but they have also suffered limitations [21,38,43,50,57,58,90]. Furthermore, a few researchers combined SSVEPs with P300 to generate more targets with less frequencies, but this was at the cost of task complexity, which eventually affected the performance of the system. In order to overcome these issues, the present study tested a hybrid approach that combines EEG and eye-tracking systems to not only reduce user discomfort but to also to achieve high classification accuracies and ITRs. The proposed framework for the implementation of the 48-target BCI speller uses only six frequencies. It is noteworthy that this is only $15 \%$ of the frequency number that is used in any BCI speller study to decode 36 or more commands to date [21,28,63]. The present BCI speller achieved an average high spelling rate of $184.06 \mathrm{bpm}$ in the cued-spelling task, and an even higher rate, $190.73 \mathrm{bpm}$, in the free-spelling task. Table 5 lists the comparison of the recently developed SSVEP-based BCI systems including the proposed approach. In this comparison, the number of electrodes used to record SSVEP, the number of commands presented, the number of frequencies used to decode these commands, the accuracy and ITR are utilized as performance evaluation metrics. To the best of our knowledge, the ITRs that were achieved by the proposed hybrid approach are not highest ever achieved, but they are among the highest achieved with any speller system (Table 5). For further comparison, the mean ITR of a code-modulated visually evoked potential (cVEP)-based system was $116.4 \mathrm{bpm}$, the mean ITR of an SSVEP-based speller was $87.50 \mathrm{bpm}$, and the mean ITR of a P300-based speller was $17.4 \mathrm{bpm} \mathrm{[28].}$ 
Table 5. Comparison of the present study with recent SSVEP-based BCI studies.

\begin{tabular}{|c|c|c|c|c|c|c|c|c|}
\hline Study & Stimuli & Multimodal & Frequency Range & NE & NC & NF & Average Accuracy (\%) & Information Transfer Rate \\
\hline Present & Rectangles & Yes & Mid & 8 & 48 & 6 & 90.35 (84.03-96.53) & 190.73 (159.23-212.31) \\
\hline Nakanishi et al. [29] & Rectangles & No & Low & 9 & 40 & 40 & 89.83 (79.50-97.50) & $325.33(263.00-376.58)$ \\
\hline Chen et al. [84] & Rectangles & No & Low & 9 & 40 & 40 & 91.95 (78.50-99.50) & $151.18(114.48-175)$ \\
\hline Chen et al. [28] & Characters & No & Low & 9 & 40 & 40 & $91.00(77.00-99.50)$ & $267.0(199.8-315.0)$ \\
\hline Bin et al. [33] & Rectangles & No & Low & 9 & 6 & 6 & $95.30(83.30-100.0)$ & $58.00(40.00-67.00)$ \\
\hline Kwak et al. [91] & LED & No & Low & 8 & 5 & 5 & $91.30(81.40-98.60)$ & $32.90(19.60-51.00)$ \\
\hline Müller -Putz et al. [92] & LED & No & Low & 4 & 4 & 4 & $72.50(44.00-88.00)$ & $19.70(4.10-34.20)$ \\
\hline Chen et al. [27] & Characters & No & Low & 9 & 45 & 45 & 88.70 (73.30-98.90) & $61.0(45.00-75.00)$ \\
\hline Martinez et al. [93] & Checkerboard & No & Low & 6 & 4 & 4 & $96.50(82.30-100.0)$ & $29.60(17.00-38.70)$ \\
\hline Min et al. [94] & Line-grid & No & Low & 3 & 6 & 6 & $42.50(20.00-63.30)$ & $3.20(0.10-9.40)$ \\
\hline
\end{tabular}

NE: number of electrodes, NC: number of commands, and NF: number of frequencies. Frequency range: low $=4-12$, mid $=12-30$, and high, $>30$. 
The present significant performance improvement can be attributed to the novel speller design that was implemented by combining the EEG and eye-tracking approaches.

The present study demonstrates the feasibility of a comfortable and high-speed speller that can achieve an ITR of up to $212.31 \mathrm{bpm}$. It should be noted here that only four subjects were familiar with the SSVEP-based BCI speller and layout. It has been reported in BCI literature that the major challenge in SSVEP-based spellers is to find a tradeoff between accuracy and ITR $[20,28,52]$. The selection of the time window has a high impact on the accuracy and ITR $[95,96]$. Many previous studies have already reported that CCA-based classification has high errors when using a short time window $[34,83,84,96]$. This might be due to the fact that decoding large number of frequencies (i.e., the number of frequencies that are equal to number of targets) may cause the production of errors in the classification of SSVEP targets. In contrast, the proposed hybrid approach uses only six frequencies to decode forty-eight targets. Furthermore, the performance of the proposed BCI-speller has been compared with the performance of a conventional SSVEP-based basic BCI-speller and a previously developed hybrid EEG and eye tracking speller. The results of this comparison revealed a significant improvement in the performance by using proposed approach (Figure 2a,b). A Mann-Whitney U test was used to statistically validate this performance improvement, and the results of this analysis showed that the proposed speller is significantly better in terms of both accuracy and ITR for all time windows with $p<0.001$. Another advantage of the proposed framework is the considerably lower probability of target-key misclassification relative to the previous BCI speller systems that use a number of frequencies equal to the number of targets (e.g., 40 targets [28]), particularly those systems that also use short-duration EEG data (e.g., 1 s) (Figure 2). As discussed above, another potential advantage is that the proposed speller has considerably low annoyingness, fatigue and tiredness as compared to the basic BCI-speller (Table 1). This can also be attributed to the use of only six frequencies, and this could also have made it possible to use the proposed speller for longer period of time which is highly difficult to do with basic BCI-spellers. In contrast, the basic speller uses forty-eight frequencies for forty-eight targets, which causes a high level of discomfort and fatigue. It was also indicated by previous studies that the simultaneous flickering of a large number of stimuli can cause discomfort and fatigue to users, and this can also affect the performance of the system. Furthermore, another important advantage of the proposed framework is that it can overcome the restrictions and limitations that are caused by the monitor refresh rate to generate large number of frequencies to decode large number of targets [34,38,39], since the proposed speller only uses six frequencies that can be generated by any monitor. In the light of the above, the proposed BCI-speller system could be used as an efficient and better alternative to the previous speller systems.

Since the main aim of this study was to propose a novel speller design for SSVEP-based BCI spellers, the proposed speller nonetheless has room for improvement. First, phase information could be added to the stimuli for more efficient target-frequency detection. Nakanishi and co-authors [20] used different phase values to better discriminate the target frequency from the recorded SSVEP signals as compared with a conventional frequency coding scheme. Additionally, the accuracy of the proposed system could be improved by using more enhanced SSVEP detection algorithms. Additionally in this regard, the algorithm that was utilized for target-frequency detection could be improved by incorporating filter bank analysis and individual training data. In filter bank analysis, all EEG data could be divided into different frequency sub-bands to improve the classification of the SSVEP targets. Chen and co-authors [84] illustrated the use of filter bank analysis to enhance the performance of conventional CCA-based target detection. Further improvements in the classification accuracy could be achieved by replacing conventional reference signals by individual training data for each target frequency. Individual training data for each target could be recorded before the actual experiment and could be used instead of sine/cosine reference signals. Yuan and co-authors [83] showed that inter-subject information could be used to update the reference signals to improve the detection of the target frequency in SSVEP-based BCIs. Moreover, the selection of flickering frequencies can be further optimized to enhance the performance of the proposed system. Figure 3 shows the confusion 
matrices for SSVEP and eye tracking predictions, which can be helpful in selecting optimal parameters for the proposed speller. It can be seen that eye tracking predictions had no errors, as the size of the target box was large enough to be detected correctly. This is consistent with the previous studies which have shown that eye tracking classifications are low if the targets are densely located with small sizes [65]. In contrast, the SSVEP predictions showed misclassifications, specifically for frequency six. The prediction errors with this frequency highly affect the overall classification accuracy and ITR of the system. Therefore, the optimization of the frequency selection could highly increase the performance of the proposed system. Furthermore, higher ITRs could be achieved if the stimulus duration was separately optimized for each subject. Indeed, system parameters such as electrode locations, the number of electrodes, stimulation frequencies, the effect of frequencies from different SSVEP ranges, and the number of trials for templates could be optimized for each individual to achieve the best system performance [97]. Therefore, it is our immediate future plan to study the effect of all these parameters on the performance of the proposed system and to select optimal parameters with best performance.

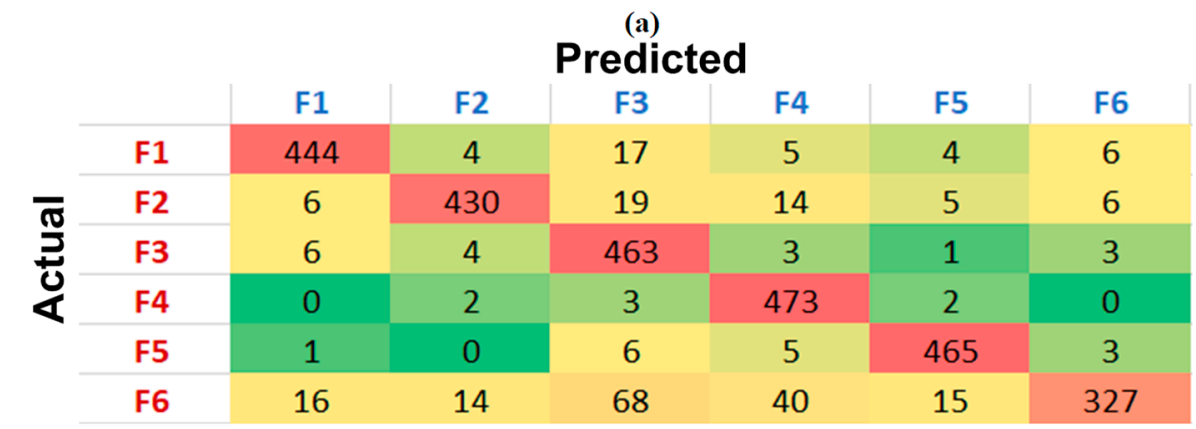

(b)

Predicted

\begin{tabular}{|c|c|c|c|c|c|c|c|c|}
\hline & Box 1 & Box 2 & Box 3 & Box 4 & Box 5 & Box 6 & Box 7 & Box 8 \\
\hline Box 1 & 360 & 0 & 0 & 0 & 0 & 0 & 0 & 0 \\
\hline Box 2 & 0 & 360 & 0 & 0 & 0 & 0 & 0 & 0 \\
\hline Box 3 & 0 & 0 & 360 & 0 & 0 & 0 & 0 & 0 \\
\hline Box 4 & 0 & 0 & 0 & 360 & 0 & 0 & 0 & 0 \\
\hline Box 5 & 0 & 0 & 0 & 0 & 360 & 0 & 0 & 0 \\
\hline Box 6 & 0 & 0 & 0 & 0 & 0 & 360 & 0 & 0 \\
\hline Box 7 & 0 & 0 & 0 & 0 & 0 & 0 & 360 & 0 \\
\hline Box 8 & 0 & 0 & 0 & 0 & 0 & 0 & 0 & 360 \\
\hline
\end{tabular}

Figure 3. Confusion matrices. (a) SSVEP predictions. (b) Eye tracking predictions.

It is important to mention here that, whereas eye-tracker-based spellers' spelling rate is 5 to 10 words per minute [28,98], they require a high sampling rate as well as a high level of user concentration on the target (i.e., "gaze control" without any movement), which is difficult for most people to manage $[65,99]$. Furthermore, the equipment needed for such eye tracking in real time is, at least at present, expensive, which makes it impractical for application [100]. One may argue that the Eyelink used in this study is also a research grade eye tracking system and can achieve good accuracy by itself. However, we argue on the basis of previous studies that have used cheap cameras for eye tracking and have shown that eye tracking classifications are low if the targets are densely located with small sizes and SSVEP-based BCI performs better in such a scenario $[65,81]$. On the other hand, there are higher chances of misclassification of targets in SSVEP-based BCIs when decoding a large number of targets. Therefore, combining eye tracking with an SSVEP-based BCI can provide a good solution that can be used to achieve best results. In other words, the present study used eye tracking to detect large boxes (a region covered by six keys, as shown in Figure 1b) that can be easily detectable by any ordinary camera, and that, therefore, do not require exceedingly high levels of concentration and motionlessness. Thus, the proposed hybrid framework can be considered for employment as an 
optimal speller not only in many BCI applications but also in many other applications like artifact rejection from EEG data.

\section{Conclusions}

This paper presents a hybrid framework to implement a novel speller design to reduce user discomfort and to increase the classification accuracy and ITR of a speller system by combining eye tracking and an SSVEP BCI with stimulus frequencies ranging from 13 to $24 \mathrm{~Hz}$. The proposed speller is superior to most of the spellers that have been developed in the literature in terms of user comfortability, items classified, classification accuracy, and ITR. The high point of the proposed speller is that it only uses six frequencies to classify forty-eight targets, whereas a basic speller uses a number of frequencies that is equal to the number of targets. Furthermore, the discomfort, fatigue, annoyingness and tiredness caused by the proposed speller are lesser as compared to the basic and hybrid spellers. A comparison with the basic and hybrid spellers revealed a statistically validated significantly better performance of the proposed framework.

Author Contributions: M.M.N.M. designed the algorithm, carried out the simulations, and analyzed the simulations results. M.M.N.M. and M.A.K. contributed to the algorithm coding, conducted the experiments and participated in data processing. S.K., H.S.C. and M.Y.J. did the literature review. M.M.N.M. and M.A.K. wrote the manuscript. M.Y.J. supervised the overall study and suggested the theoretical aspects of the study. All authors have approved the final manuscript. All authors provided critical feedback on the manuscript. All authors have read and agreed to the published version of the manuscript.

Funding: This work was supported by a National Research Foundation of Korea (NRF) grant funded by the Korean government (MSIP) (NO.2015R1A5A1037668) and (NO.2017R1A2B2006999).

Acknowledgments: The authors would like to thank the study participants for their participation and for their feedback.

Conflicts of Interest: The authors declare no conflict of interest.

Availability of Data and Materials: The dataset used and/or analyzed during the current study are available from the corresponding author upon reasonable request.

Ethics Approval and Consent to Participate: The study was approved by the Institutional Review Board of Pusan National University and all experiments were carried out in accordance with the approved study protocol. The subjects provided written informed consent before participation and consented to the publishing of their collected data.

\section{References}

1. Vidal, J.J. Toward direct brain-computer communication. Annu. Rev. Biophys. Bioeng. 1973, 2, $157-180$. [CrossRef] [PubMed]

2. Vidal, J.J. Real-time detection of brain events in EEG. Proc. IEEE 1977, 65, 633-641. [CrossRef]

3. Bozinovski, S.; Sestakov, M.; Bozinovska, L. Using EEG alpha rhythm to control a mobile robot. In Proceedings of the Annual International Conference of the IEEE Engineering in Medicine and Biology Society, New Orleans, LA, USA, 4-7 November 1988; pp. 1515-1516.

4. Farwell, L.A.; Donchin, E. Talking off the top of your head: Toward a mental prosthesis utilizing event-related brain potentials. Electroencephalogr. Clin. Neurophysiol. 1988, 70, 510-523. [CrossRef]

5. Bozinovski, S.; Bozinovski, A. Mental states, EEG manifestations, and mentally emulated digital circuits for brain-robot interaction. IEEE Trans. Auton. Ment. Dev. 2015, 7, 39-51. [CrossRef]

6. Li, Y.; Pan, J.; Long, J.; Yu, T.; Wang, F.; Yu, Z.; Wu, W. Multimodal BCIs: Target detection, multidimensional control, and awareness evaluation in patients with disorder of consciousness. Proc. IEEE 2016, 104, 332-352.

7. Wolpaw, J.R.; Birbaumer, N.; McFarland, D.J.; Pfurtscheller, G.; Vaughan, T.M. Brain-computer interfaces for communication and control. Clin. Neurophysiol. 2002, 113, 767-791. [CrossRef]

8. Xie, J.; Xu, G.; Luo, A.; Li, M.; Zhang, S.; Han, C.; Yan, W. The Role of Visual Noise in Influencing Mental Load and Fatigue in a Steady-State Motion Visual Evoked Potential-Based Brain-Computer Interface. Sensors 2017, 17, 1873. [CrossRef]

9. Ahn, M.; Cho, H.; Ahn, S.; Jun, S.C. User's Self-Prediction of Performance in Motor Imagery Brain-Computer Interface. Front. Hum. Neurosci. 2018, 12, 59. [CrossRef] 
10. Liu, Y.-H.; Huang, S.; Huang, Y.-D. Motor Imagery EEG Classification for Patients with Amyotrophic Lateral Sclerosis Using Fractal Dimension and Fisher's Criterion-Based Channel Selection. Sensors 2017, 17, 1557. [CrossRef]

11. Lazarou, I.; Nikolopoulos, S.; Petrantonakis, P.C.; Kompatsiaris, I.; Tsolaki, M. EEG-Based Brain-Computer Interfaces for Communication and Rehabilitation of People with Motor Impairment: A Novel Approach of the 21st Century. Front. Hum. Neurosci. 2018, 12, 14. [CrossRef]

12. Shin, J.; Kwon, J.; Im, C.-H. A Ternary Hybrid EEG-NIRS Brain-Computer Interface for the Classification of Brain Activation Patterns during Mental Arithmetic, Motor Imagery, and Idle State. Front. Neuroinform. 2018, 12, 5. [CrossRef] [PubMed]

13. Kamran, M.A.; Mannan, M.M.N.; Jeong, M.Y. Drowsiness, Fatigue and Poor Sleep's Causes and Detection: A Comprehensive Study. IEEE Access 2019, 7, 167172-167186. [CrossRef]

14. Amiri, S.; Fazel-Rezai, R.; Asadpour, V. A review of hybrid brain-computer interface systems. Adv. Hum. -Comput. Interact. 2013, 2013, 1. [CrossRef]

15. Floriano, A.; F Diez, P.; Freire Bastos-Filho, T. Evaluating the Influence of Chromatic and Luminance Stimuli on SSVEPs from Behind-the-Ears and Occipital Areas. Sensors 2018, 18, 615. [CrossRef]

16. Blankertz, B.; Lemm, S.; Treder, M.; Haufe, S.; Müller, K.-R. Single-trial analysis and classification of ERP components-A tutorial. NeuroImage 2011, 56, 814-825. [CrossRef]

17. Bozinovska, L.; Bozinovski, S.; Stojanov, G. Electroexpectogram: Experimental Design and Agorithms. In Proceedings of the 1992 International Biomedical Engineering Days, Istanbul, Turkey, 18-20 August 1992; pp. 55-60.

18. Birbaumer, N.; Ghanayim, N.; Hinterberger, T.; Iversen, I.; Kotchoubey, B.; Kübler, A.; Perelmouter, J.; Taub, E.; Flor, H. A spelling device for the paralysed. Nature 1999, 398, 297. [CrossRef]

19. Xu, M.; Chen, L.; Zhang, L.; Qi, H.; Ma, L.; Tang, J.; Wan, B.; Ming, D. A visual parallel-BCI speller based on the time-frequency coding strategy. J. Neural Eng. 2014, 11, 026014. [CrossRef]

20. Nakanishi, M.; Wang, Y.; Wang, Y.-T.; Jung, T.-P. A comparison study of canonical correlation analysis based methods for detecting steady-state visual evoked potentials. PLoS ONE 2015, 10, e0140703. [CrossRef]

21. Won, D.-O.; Hwang, H.-J.; Dähne, S.; Müller, K.-R.; Lee, S.-W. Effect of higher frequency on the classification of steady-state visual evoked potentials. J. Neural Eng. 2015, 13, 016014. [CrossRef]

22. Yin, E.; Zeyl, T.; Saab, R.; Chau, T.; Hu, D.; Zhou, Z. A hybrid brain-computer interface based on the fusion of P300 and SSVEP scores. IEEE Trans. Neural Syst. Rehabil. Eng. 2015, 23, 693-701. [CrossRef]

23. Wang, Y.; Chen, X.; Gao, X.; Gao, S. A Benchmark Dataset for SSVEP-Based Brain-Computer Interfaces. IEEE Trans. Neural Syst. Rehabil. Eng. 2017, 25, 1746-1752. [CrossRef]

24. Won, D.-O.; Hwang, H.-J.; Kim, D.-M.; Müller, K.-R.; Lee, S.-W. Motion-Based Rapid Serial Visual Presentation for Gaze-Independent Brain-Computer Interfaces. IEEE Trans. Neural Syst. Rehabil. Eng. 2018, 26, 334-343. [CrossRef]

25. Cecotti, H. A self-paced and calibration-less SSVEP-based brain-computer interface speller. IEEE Trans. Neural Syst. Rehabil. Eng. 2010, 18, 127-133. [CrossRef]

26. Kimura, Y.; Tanaka, T.; Higashi, H.; Morikawa, N. SSVEP-based brain-computer interfaces using FSK-modulated visual stimuli. IEEE Trans. Biomed. Eng. 2013, 60, 2831-2838. [CrossRef]

27. Chen, X.; Chen, Z.; Gao, S.; Gao, X. A high-itr ssvep-based bci speller. Brain-Comput. Interfaces 2014, 1, 181-191. [CrossRef]

28. Chen, X.; Wang, Y.; Nakanishi, M.; Gao, X.; Jung, T.-P.; Gao, S. High-speed spelling with a noninvasive brain-computer interface. Proc. Natl. Acad. Sci. USA 2015, 112, E6058-E6067. [CrossRef]

29. Nakanishi, M.; Wang, Y.; Chen, X.; Wang, Y.-T.; Gao, X.; Jung, T.-P. Enhancing Detection of SSVEPs for a high-speed brain speller using task-related component analysis. IEEE Trans. Biomed. Eng. 2018, 65, 104-112. [CrossRef]

30. Volosyak, I. SSVEP-based Bremen-BCI interface-boosting information transfer rates. J. Neural Eng. 2011, 8, 036020. [CrossRef]

31. Spüler, M.; Rosenstiel, W.; Bogdan, M. Online adaptation of a c-VEP brain-computer interface (BCI) based on error-related potentials and unsupervised learning. PLoS ONE 2012, 7, e51077. [CrossRef]

32. Gembler, F.; Stawicki, P.; Volosyak, I. Autonomous parameter adjustment for SSVEP-based BCIs with a novel BCI wizard. Front. Neurosci. 2015, 9, 474. [CrossRef] 
33. Bin, G.; Gao, X.; Yan, Z.; Hong, B.; Gao, S. An online multi-channel SSVEP-based brain-computer interface using a canonical correlation analysis method. J. Neural Eng. 2009, 6, 046002. [CrossRef] [PubMed]

34. Nakanishi, M.; Wang, Y.; Wang, Y.-T.; Mitsukura, Y.; Jung, T.-P. Generating visual flickers for eliciting robust steady-state visual evoked potentials at flexible frequencies using monitor refresh rate. PLoS ONE 2014, 9, e99235. [CrossRef] [PubMed]

35. Nakanishi, M.; Wang, Y.; Wang, Y.-T.; Mitsukura, Y.; Jung, T.-P. A high-speed brain speller using steady-state visual evoked potentials. Int. J. Neural Syst. 2014, 24, 1450019. [CrossRef] [PubMed]

36. Yin, E.; Zhou, Z.; Jiang, J.; Yu, Y.; Hu, D. A dynamically optimized SSVEP brain-computer interface (BCI) speller. IEEE Trans. Biomed. Eng. 2015, 62, 1447-1456. [CrossRef]

37. Maye, A.; Zhang, D.; Engel, A.K. Utilizing retinotopic mapping for a multi-target SSVEP BCI with a single flicker frequency. IEEE Trans. Neural Syst. Rehabil. Eng. 2017, 25, 1026-1036. [CrossRef]

38. Chen, X.; Wang, Y.; Zhang, S.; Gao, S.; Hu, Y.; Gao, X. A novel stimulation method for multi-class SSVEP-BCI using intermodulation frequencies. J. Neural Eng. 2017, 14, 026013. [CrossRef]

39. Andersen, S.K.; Müller, M.M. Driving steady-state visual evoked potentials at arbitrary frequencies using temporal interpolation of stimulus presentation. BMC Neurosci. 2015, 16, 95. [CrossRef]

40. Müller-Putz, G.R.; Scherer, R.; Brauneis, C.; Pfurtscheller, G. Steady-state visual evoked potential (SSVEP)-based communication: Impact of harmonic frequency components. J. Neural Eng. 2005, 2, 123. [CrossRef]

41. Lee, P.-L.; Yeh, C.-L.; Cheng, J.Y.-S.; Yang, C.-Y.; Lan, G.-Y. An SSVEP-based BCI using high duty-cycle visual flicker. IEEE Trans. Biomed. Eng. 2011, 58, 3350-3359. [CrossRef]

42. Bakardjian, H.; Tanaka, T.; Cichocki, A. Optimization of SSVEP brain responses with application to eight-command Brain-Computer Interface. Neurosci. Lett. 2010, 469, 34-38. [CrossRef] [PubMed]

43. Sakurada, T.; Kawase, T.; Komatsu, T.; Kansaku, K. Use of high-frequency visual stimuli above the critical flicker frequency in a SSVEP-based BMI. Clin. Neurophysiol. 2015, 126, 1972-1978. [CrossRef] [PubMed]

44. Lee, P.-L.; Sie, J.-J.; Liu, Y.-J.; Wu, C.-H.; Lee, M.-H.; Shu, C.-H.; Li, P.-H.; Sun, C.-W.; Shyu, K.-K. An SSVEP-actuated brain computer interface using phase-tagged flickering sequences: A cursor system. Ann. Biomed. Eng. 2010, 38, 2383-2397. [CrossRef] [PubMed]

45. Jia, C.; Gao, X.; Hong, B.; Gao, S. Frequency and phase mixed coding in SSVEP-based brain-computer interface. IEEE Trans. Biomed. Eng. 2011, 58, 200-206. [PubMed]

46. Chien, Y.-Y.; Lin, F.-C.; Zao, J.K.; Chou, C.-C.; Huang, Y.-P.; Kuo, H.-Y.; Wang, Y.; Jung, T.-P.; Shieh, H.-P.D. Polychromatic SSVEP stimuli with subtle flickering adapted to brain-display interactions. J. Neural Eng. 2017, 14, 016018. [CrossRef]

47. Shyu, K.-K.; Lee, P.-L.; Liu, Y.-J.; Sie, J.-J. Dual-frequency steady-state visual evoked potential for brain computer interface. Neurosci. Lett. 2010, 483, 28-31. [CrossRef]

48. Hwang, H.-J.; Kim, D.H.; Han, C.-H.; Im, C.-H. A new dual-frequency stimulation method to increase the number of visual stimuli for multi-class SSVEP-based brain-computer interface (BCI). Brain Res. 2013, 1515, 66-77. [CrossRef]

49. Wang, Y.; Jung, T.-p. Visual stimulus design for high-rate SSVEP BCI. Electron. Lett. 2010, 46, 1057-1058. [CrossRef]

50. Zhang, Y.; Xu, P.; Liu, T.; Hu, J.; Zhang, R.; Yao, D. Multiple frequencies sequential coding for SSVEP-based brain-computer interface. PLoS ONE 2012, 7, e29519. [CrossRef]

51. Lopez-Gordo, M.; Prieto, A.; Pelayo, F.; Morillas, C. Use of phase in brain-computer interfaces based on steady-state visual evoked potentials. Neural Process. Lett. 2010, 32, 1-9. [CrossRef]

52. Chang, M.H.; Baek, H.J.; Lee, S.M.; Park, K.S. An amplitude-modulated visual stimulation for reducing eye fatigue in SSVEP-based brain-computer interfaces. Clin. Neurophysiol. 2014, 125, 1380-1391. [CrossRef]

53. Chen, X.; Chen, Z.; Gao, S.; Gao, X. Brain-computer interface based on intermodulation frequency. J. Neural Eng. 2013, 10, 066009. [CrossRef] [PubMed]

54. Zhang, X.; Xu, G.; Xie, J.; Zhang, X. Brain response to luminance-based and motion-based stimulation using inter-modulation frequencies. PLOS ONE 2017, 12, e0188073. [CrossRef] [PubMed]

55. Shyu, K.-K.; Chiu, Y.-J.; Lee, P.-L.; Liang, J.-M.; Peng, S.-H. Adaptive SSVEP-based BCI system with frequency and pulse duty-cycle stimuli tuning design. IEEE Trans. Neural Syst. Rehabil. Eng. 2013, 21, 697-703. [CrossRef] 
56. Diez, P.F.; Müller, S.M.T.; Mut, V.A.; Laciar, E.; Avila, E.; Bastos-Filho, T.F.; Sarcinelli-Filho, M. Commanding a robotic wheelchair with a high-frequency steady-state visual evoked potential based brain-computer interface. Med. Eng. Phys. 2013, 35, 1155-1164. [CrossRef]

57. Oralhan, Z.; Tokmakci, M. The Effect of Duty Cycle and Brightness Variation of Visual Stimuli on SSVEP in Brain Computer Interface Systems. IETE J. Res. 2016, 62, 795-803. [CrossRef]

58. Mouli, S.; Palaniappan, R. Toward a reliable PWM-based light-emitting diode visual stimulus for improved SSVEP response with minimal visual fatigue. J. Eng. 2017, 1. [CrossRef]

59. Xu, M.; Qi, H.; Wan, B.; Yin, T.; Liu, Z.; Ming, D. A hybrid BCI speller paradigm combining P300 potential and the SSVEP blocking feature. J. Neural Eng. 2013, 10, 026001. [CrossRef]

60. Yin, E.; Zhou, Z.; Jiang, J.; Chen, F.; Liu, Y.; Hu, D. A novel hybrid BCI speller based on the incorporation of SSVEP into the P300 paradigm. J. Neural Eng. 2013, 10, 026012. [CrossRef]

61. Wang, M.; Daly, I.; Allison, B.Z.; Jin, J.; Zhang, Y.; Chen, L.; Wang, X. A new hybrid BCI paradigm based on P300 and SSVEP. J. Neurosci. Methods 2015, 244, 16-25. [CrossRef]

62. Chang, M.H.; Lee, J.S.; Heo, J.; Park, K.S. Eliciting dual-frequency SSVEP using a hybrid SSVEP-P300 BCI. J. Neurosci. Methods 2016, 258, 104-113. [CrossRef]

63. Yin, E.; Zhou, Z.; Jiang, J.; Chen, F.; Liu, Y.; Hu, D. A speedy hybrid BCI spelling approach combining P300 and SSVEP. IEEE Trans. Biomed. Eng. 2014, 61, 473-483. [PubMed]

64. Lin, K.; Cinetto, A.; Wang, Y.; Chen, X.; Gao, S.; Gao, X. An online hybrid BCI system based on SSVEP and EMG. J. Neural Eng. 2016, 13, 026020. [CrossRef] [PubMed]

65. Suefusa, K.; Tanaka, T. A comparison study of visually stimulated brain-computer and eye-tracking interfaces. J. Neural Eng. 2017, 14, 036009. [CrossRef] [PubMed]

66. Bozinovski, S. Mobile robot trajectory control: From fixed rails to direct bioelectric control. In Proceedings of the IEEE International Workshop on Intelligent Motion Control, Istanbul, Turkey, 20-22 August 1990; pp. 463-467.

67. Al-Rahayfeh, A.; Faezipour, M. Eye tracking and head movement detection: A state-of-art survey. IEEE J. Transl. Eng. Health Med. 2013, 1, 2100212. [CrossRef]

68. Caligari, M.; Godi, M.; Guglielmetti, S.; Franchignoni, F.; Nardone, A. Eye tracking communication devices in amyotrophic lateral sclerosis: Impact on disability and quality of life. Amyotroph. Lateral Scler. Front. Degener. 2013, 14, 546-552. [CrossRef]

69. Hwang, C.-S.; Weng, H.-H.; Wang, L.-F.; Tsai, C.-H.; Chang, H.-T. An eye-tracking assistive device improves the quality of life for ALS patients and reduces the caregivers' burden. J. Mot. Behav. 2014, 46, 233-238. [CrossRef]

70. Majaranta, P.; Bulling, A. Eye tracking and eye-based human-computer interaction. In Advances in Physiological Computing; Springer: London, UK, 2014; pp. 39-65.

71. Käthner, I.; Kübler, A.; Halder, S. Comparison of eye tracking, electrooculography and an auditory brain-computer interface for binary communication: A case study with a participant in the locked-in state. J. Neuroeng. Rehabil. 2015, 12, 76. [CrossRef]

72. Pasqualotto, E.; Matuz, T.; Federici, S.; Ruf, C.A.; Bartl, M.; Olivetti Belardinelli, M.; Birbaumer, N.; Halder, S. Usability and workload of access technology for people with severe motor impairment: A comparison of brain-computer interfacing and eye tracking. Neurorehabilit. Neural Repair 2015, 29, 950-957. [CrossRef]

73. Popa, L.; Selejan, O.; Scott, A.; Mureşanu, D.F.; Balea, M.; Rafila, A. Reading beyond the glance: Eye tracking in neurosciences. Neurol. Sci. 2015, 36, 683-688. [CrossRef]

74. Mannan, M.M.N.; Kim, S.; Jeong, M.Y.; Kamran, M.A. Hybrid EEG—Eye tracker: Automatic identification and removal of eye movement and blink artifacts from electroencephalographic signal. Sensors 2016, 16, 241. [CrossRef]

75. Mannan, M.M.N.; Jeong, M.Y.; Kamran, M.A. Hybrid ICA—Regression: Automatic identification and removal of ocular artifacts from electroencephalographic signals. Front. Hum. Neurosci. 2016, 10, 193. [CrossRef]

76. Yong, X.; Fatourechi, M.; Ward, R.K.; Birch, G.E. The design of a point-and-click system by integrating a self-paced brain-computer interface with an Eye-tracker. IEEE J. Emerg. Sel. Top. Circuits Syst. 2011, 1, 590-602. [CrossRef]

77. Yong, X.; Fatourechi, M.; Ward, R.K.; Birch, G.E. Automatic artefact removal in a self-paced hybrid brain-computer interface system. J. Neuroeng. Rehabil. 2012, 9, 50. [CrossRef] [PubMed] 
78. McMullen, D.P.; Hotson, G.; Katyal, K.D.; Wester, B.A.; Fifer, M.S.; McGee, T.G.; Harris, A.; Johannes, M.S.; Vogelstein, R.J.; Ravitz, A.D. Demonstration of a semi-autonomous hybrid brain-machine interface using human intracranial EEG, eye tracking, and computer vision to control a robotic upper limb prosthetic. IEEE Trans. Neural Syst. Rehabil. Eng. 2014, 22, 784-796. [CrossRef] [PubMed]

79. Stawicki, P.; Gembler, F.; Rezeika, A.; Volosyak, I. A novel hybrid mental spelling application based on eye tracking and SSVEP-based BCI. Brain Sci. 2017, 7, 35. [CrossRef]

80. McCullagh, P.; Brennan, C.; Lightbody, G.; Galway, L.; Thompson, E.; Martin, S. An SSVEP and eye tracking hybrid BNCI: Potential beyond communication and control. In Proceedings of the International Conference on Augmented Cognition, Toronto, ON, Canada, 17-22 July 2016; pp. 69-78.

81. Lim, J.-H.; Lee, J.-H.; Hwang, H.-J.; Kim, D.H.; Im, C.-H. Development of a hybrid mental spelling system combining SSVEP-based brain-computer interface and webcam-based eye tracking. Biomed. Signal Process. Control 2015, 21, 99-104. [CrossRef]

82. Cao, T.; Wan, F.; Wong, C.M.; da Cruz, J.N.; Hu, Y. Objective evaluation of fatigue by EEG spectral analysis in steady-state visual evoked potential-based brain-computer interfaces. Biomed. Eng. Online 2014, 13, 28. [CrossRef]

83. Yuan, P.; Chen, X.; Wang, Y.; Gao, X.; Gao, S. Enhancing performances of SSVEP-based brain-computer interfaces via exploiting inter-subject information. J. Neural Eng. 2015, 12, 046006. [CrossRef]

84. Chen, X.; Wang, Y.; Gao, S.; Jung, T.-P.; Gao, X. Filter bank canonical correlation analysis for implementing a high-speed SSVEP-based brain-computer interface. J. Neural Eng. 2015, 12, 046008. [CrossRef]

85. Tian, Y.; Li, F.; Xu, P.; Yuan, Z.; Zhao, D.; Zhang, H. Combining canonical correlation analysis and infinite reference for frequency recognition of steady-state visual evoked potential recordings: A comparison with periodogram method. Bio-Med Mater. Eng. 2014, 24, 2901-2908. [CrossRef]

86. Manyakov, N.V.; Chumerin, N.; Van Hulle, M.M. Multichannel decoding for phase-coded SSVEP brain-computer interface. Int. J. Neural Syst. 2012, 22, 1250022. [CrossRef] [PubMed]

87. Carvalho, S.N.; Costa, T.B.; Uribe, L.F.; Soriano, D.C.; Yared, G.F.; Coradine, L.C.; Attux, R. Comparative analysis of strategies for feature extraction and classification in SSVEP BCIs. Biomed. Signal Process. Control 2015, 21, 34-42. [CrossRef]

88. Lin, Z.; Zhang, C.; Wu, W.; Gao, X. Frequency recognition based on canonical correlation analysis for SSVEP-based BCIs. IEEE Trans. Biomed. Eng. 2006, 53, 2610-2614. [CrossRef] [PubMed]

89. Lim, J.H.; Kim, Y.W.; Lee, J.H.; An, K.O.; Hwang, H.J.; Cha, H.S.; Han, C.H.; Im, C.H. An emergency call system for patients in locked-in state using an SSVEP-based brain switch. Psychophysiology 2017, 54, 1632-1643. [CrossRef] [PubMed]

90. Kramberger, I.; Donaj, G. Binocular Phase-Coded Visual Stimuli for SSVEP-Based BCI. IEEE Access 2019, 7, 48912-48922. [CrossRef]

91. Kwak, N.-S.; Müller, K.-R.; Lee, S.-W. A lower limb exoskeleton control system based on steady state visual evoked potentials. J. Neural Eng. 2015, 12, 056009. [CrossRef]

92. Muller-Putz, G.R.; Pfurtscheller, G. Control of an electrical prosthesis with an SSVEP-based BCI. IEEE Trans. Biomed. Eng. 2008, 55, 361-364. [CrossRef]

93. Martinez, P.; Bakardjian, H.; Cichocki, A. Fully online multicommand brain-computer interface with visual neurofeedback using SSVEP paradigm. Comput. Intell. Neurosci. 2007, 2007. [CrossRef]

94. Min, B.-K.; Dähne, S.; Ahn, M.-H.; Noh, Y.-K.; Müller, K.-R. Decoding of top-down cognitive processing for SSVEP-controlled BMI. Sci. Rep. 2016, 6, 36267. [CrossRef]

95. Liu, Q.; Chen, K.; Ai, Q.; Xie, S.Q. recent development of signal processing algorithms for SSVEP-based brain computer interfaces. J. Med. Biol. Eng. 2014, 34, 299-309. [CrossRef]

96. Zhang, Y.; Zhou, G.; Jin, J.; Wang, X.; Cichocki, A. Frequency recognition in SSVEP-based BCI using multiset canonical correlation analysis. Int. J. Neural Syst. 2014, 24, 1450013. [CrossRef] [PubMed]

97. Carvalho, S.N.; Costa, T.B.; Uribe, L.F.; Soriano, D.C.; Almeida, S.R.; Min, L.L.; Castellano, G.; Attux, R. Effect of the combination of different numbers of flickering frequencies in an SSVEP-BCI for healthy volunteers and stroke patients. In Proceedings of the 2015 7th International IEEE/EMBS Conference onNeural Engineering (NER), Montpellier, France, 22-24 April 2015; pp. 78-81.

98. Majaranta, P.; Räihä, K.-J. Twenty years of eye typing: Systems and design issues. In Proceedings of the 2002 Symposium on Eye Tracking Research \& Applications, New Orleans, LA, USA, 25-27 March 2002; pp. 15-22. 
99. Dybdal, M.L.; Agustin, J.S.; Hansen, J.P. Gaze input for mobile devices by dwell and gestures. In Proceedings of the Symposium on Eye Tracking Research and Applications 2012, Santa Barbara, CA, USA, 28-30 March 2012; pp. 225-228.

100. Lee, H.C.; Lee, W.O.; Cho, C.W.; Gwon, S.Y.; Park, K.R.; Lee, H.; Cha, J. Remote gaze tracking system on a large display. Sensors 2013, 13, 13439-13463. [CrossRef] [PubMed] 